Bull. Austral. Math. Soc.

$53 \mathrm{~A} 04,53 \mathrm{~A} 05$

VoL. 65 (2002) [73-78]

\title{
TOTAL TORSION OF CLOSED LINES OF CURVATURE
}

\author{
YONG-AN QIN AND SHI-JIE LI
}

In'this article we investigate the total torsion of closed lines of curvature on a surface in $\mathbb{E}^{3}$ and obtain the following results.

(1) The total torsion of a closed line of curvature on a surface is $k \pi$, where $k$ is an integer. Conversely, if the total torsion of a closed curve is $k \pi$ for an integer $k$, then the curve can appear as a line of curvature on a surface. In particular, if the total torsion of a closed curve is $2 k \pi$, then it can appear as a line of curvature on a closed, oriented surface of genus 1 .

(2) The total torsion of a closed line of curvature on an ovaloid is zero.

\section{INTRODUCTION}

Let $C$ be a closed curve in three dimensional Euclidean space $\mathbb{E}^{3}$. One of the global properties of $C$ is its total torsion $T$ defined by the integral $T=\int_{C} \tau d s$, where $s$ and $\tau$ are the arc length and the torsion of $C$, respectively. It is well known that for any real number $r$ there is a closed curve $C$ such that its total torsion $T$ is equal to $r$. On the other hand, we have the following a theorem of Geppert [3] (see for instance, [4]).

THEOREM A. The total torsion of a closed curve on a unit sphere is zero.

In [2] Chen investigated the total torsion of a class of closed curves on a developable surface and proved the following theorem.

Theorem B. Let $C$ be a closed curve on a developable surface $M$. If $C$ is perpendicular to the rectilinear generators of $M$ everywhere, then the total torsion of $C$ is zero.

In this article, we investigate the total torsion of closed lines of curvature on a surface and particularly on an ovaloid and obtain the following results.

Received 14th May, 2001

The first author was supported by NSFC (Project 19771039) of China and the second by PNSF (Project 960179) of Guangdong Province, China.

The authors would like to express their gratitude to Professor W. Klingenberg and Professor Y. Hong for their many valuable comments and suggestions.

Copyright Clearance Centre, Inc. Serial-fee code: 0004-9727/02 \$A2.00+0.00. 
THEOREM 1. The total torsion of a closed line of curvature on a surface in $\mathbb{E}^{3}$ is $k \pi$, where $k$ is an integer. Conversely, if the total torsion of a closed curve in $\mathbb{E}^{3}$ is $k \pi$ for an integer $k$, then the curve can appear as a line of curvature on a surface. In particularly, if the total torsion of a closed curve is $2 k \pi$, then it can appear as a line of curvature on a closed, oriented surface of genus 1.

THEOREM 2. The total torsion of a closed line of curvature on an ovaloid in $\mathbb{E}^{3}$ is zero.

From our results, we can see that Theorem 1 and Theorem 2 generalise Theorem $\mathrm{B}$ and Theorem A, respectively, since the curve in Theorem B is actually a line of curvature on a developable surface and the sphere is an ovaloid on which any curve is a line of curvature.

In [1], Blaschke proposed 16 problems on the differential geometry of ovaloids, the first one among them is: What can we say about the lines of curvature on an ovaloid? Theorem 2 is a partial response to the problem.

\section{Proofs of Theorems}

Let $C: \mathbf{r}=\mathbf{r}(s), 0 \leqslant s \leqslant L$, be a closed curve on a surface $M$ in $\mathbb{E}^{3}$, where $s$ is the arc length of $C$. Suppose that $C$ has no point with $\ddot{\mathbf{r}}=0$. Denote the Frenet frame of $C$ by $\{\mathbf{r} ; \boldsymbol{\alpha}, \beta, \gamma\}$, where $\alpha=\dot{\mathbf{r}}, \beta=\ddot{\mathbf{r}} /|\ddot{\mathbf{r}}|$, and $\gamma=\alpha \times \beta$. Denote the Darboux frame of $C$ on $M$ by $\{\mathbf{r} ; \boldsymbol{\alpha}, \boldsymbol{\nu}, \mathbf{n}\}$, where $\mathbf{n}=\mathbf{n}(s)=\mathbf{n}(\mathbf{r}(s))$ is the normal vector field on $M$ along $C$ and $\nu=\mathbf{n} \times \boldsymbol{\alpha}$. Since the Darboux frame is orthonormal, we have the following equations:

$$
\left\{\begin{array}{l}
\dot{\alpha}=\quad k_{g} \nu+k_{n} \mathbf{n}, \\
\dot{\nu}=-k_{g} \alpha \quad+\tau_{g} \mathbf{n} \\
\dot{\mathbf{n}}=-k_{n} \boldsymbol{\alpha}-\tau_{g} \nu
\end{array}\right.
$$

where the functions $k_{g}$ and $k_{n}$ are the geodesic curvature and normal curvature of $C$, respectively, and the function $\tau_{g}$ is the geodesic torsion of $C$.

Choosing $\{\nu(s), \mathbf{n}(s)\}$ as the positive orientation of the plane spanned by $\nu(s)$ and $\mathbf{n}(s)$, we define $\theta(s)$ to be the oriented angle from $\boldsymbol{\beta}(s)$ to $\mathbf{n}(s)$, then we have

$$
\mathbf{n}(s)=\beta(s) \cos \theta(s)+\gamma(s) \sin \theta(s)
$$

and

$$
\nu(s)=\mathbf{n}(s) \times \boldsymbol{\alpha}(s)=\boldsymbol{\beta}(s) \sin \theta(s)-\gamma(s) \cos \theta(s) .
$$

From (1), (2) and (3), with the help of the Frenet equations, we find that the geodesic torsion $\tau_{g}$ of $C$ satisfies the following equation.

$$
\tau_{g}=\langle\dot{\nu}, \mathbf{n}\rangle=\tau+\dot{\theta}
$$


where $\dot{\nu}=d \nu / d s$ and $\dot{\theta}=d \theta / d s$.

If $C$ is a line of curvature on a surface $M$, then by the Rodrigues' formula, (that is, $\dot{\mathbf{n}}=-\kappa \boldsymbol{\alpha}$, where the function $\kappa$ is the curvature of $C$ ), we have the following

$$
\tau_{g}=-\langle\dot{\mathbf{n}}, \nu\rangle=\langle\kappa \alpha, \nu\rangle=0 .
$$

It is clear that the converse is also true. Thus we have the following (see for instance, [5]).

Lemma 1. A curve $C$ on a surface $M$ is a line of curvature on $M$ if and only if the geodesic torsion $\tau_{g}$ of $C$ vanishes.

Proof of Theorem 1: Let $C: \mathbf{r}=\mathbf{r}(s), 0 \leqslant s \leqslant L$, be a closed line of curvature on a surface $M$ in $\mathbb{E}^{3}$, where $s$ is the arc length of $C$. Then by Lemma 1 and (4) we have

$$
\tau=-\dot{\theta}
$$

thus we have

$$
T=\int_{C} \tau d s=-\int_{0}^{L} \dot{\theta} d s=\theta(0)-\theta(L) .
$$

Since $C$ is closed, $\mathbf{r}(0)=\mathbf{r}(L)$ and $\mathbf{r}^{(k)}(0)=\mathbf{r}^{(k)}(L)$, for $k=1,2,3$, where $\mathbf{r}^{(k)}=d^{k} \mathbf{r} / d s^{k}$. Consequently, we have

$$
\begin{aligned}
& \boldsymbol{\alpha}(0)=\dot{\mathbf{r}}(0)=\dot{\mathbf{r}}(L)=\boldsymbol{\alpha}(L), \\
& \beta(0)=\frac{\ddot{\mathbf{r}}(0)}{|\ddot{\mathbf{r}}(0)|}=\frac{\ddot{\mathbf{r}}(L)}{|\ddot{\mathbf{r}}(L)|}=\boldsymbol{\beta}(L) .
\end{aligned}
$$

Since $\mathbf{n}(0)$ is equal to either $\mathbf{n}(L)$ or $-\mathbf{n}(L)$, we may have

$$
T=\theta(0)-\theta(L)=k \pi,
$$

for some integer $k$.

Conversely, suppose that $C$ is a closed curve with total torsion $k \pi$, where $k$ is an integer. Denote the Frenet frame of $C$ by $\{\mathbf{r} ; \boldsymbol{\alpha}, \boldsymbol{\beta}, \boldsymbol{\gamma}\}$. We define a unit vector field $\mathbf{n}(s)$ along $C$ as follows.

$$
\mathbf{n}(s)=\beta(s) \cos \phi(s)+\gamma(s) \sin \phi(s),
$$

where $\phi(s)$ is defined by

$$
\phi(s)=-\int_{0}^{s} \tau(s) d s
$$


Then we construct the following parameterised surface $S_{1}$.

$$
\mathbf{r}_{1}=\mathbf{r}_{1}(s, t)=\mathbf{r}(s)+\operatorname{tn}(s) \times \alpha(s),|t|<\varepsilon, 0 \leqslant s \leqslant L .
$$

Since we have $\alpha(0)=\alpha(L)$ and $\beta(0)=\beta(L)$ such as above, then from (11) and the hypothersis, we have

$$
\phi(0)=0, \phi(L)=-\int_{0}^{L} \tau d s=-k \pi
$$

thus we obtain $\mathbf{n}(0)= \pm \mathbf{n}(L)$.

Now, since $C$ is closed, there is a small positive number $\varepsilon$ such that $S_{1}$ is a regular surface and $\mathbf{n}(s)$ is the normal unit vetor field of $S_{1}$ along $C$ and also $\phi$ is equal to $\theta$ in (2). Differentiating (11), we obtain

$$
\dot{\theta}(s)+\tau(s)=0
$$

from which we may conclude that $C$ is a line of curvature on $M$, according to (4) and Lemma 1.

Moreover if, in particular, $C$ is a closed curve with total torsion $2 k \pi$, where $k$ is an integer, then we may construct the following parameterised surface $S_{2}$.

$$
\begin{gathered}
\mathbf{r}_{2}=\mathbf{r}_{2}(s, t)=\mathbf{r}(s)+\delta[\mathbf{n}(s)+\mathbf{n}(s) \times \boldsymbol{\alpha}(s) \cos t+\mathbf{n}(s) \sin t] \\
0 \leqslant t<2 \pi, 0 \leqslant s \leqslant L
\end{gathered}
$$

where $\delta$ is a constant. Then since the total torsion of $C$ is equal to $2 k \pi$, we have with the help of (10) and (11)

$$
\mathbf{r}_{2}(0, t)=\mathbf{r}_{2}(L, t), 0 \leqslant t<2 \pi .
$$

Now it is easy to see that $S_{2}$ is a torus and $C$ is on $S_{2}$ with $t=3 \pi / 2$ if it is a regular surface.

To prove that $S_{2}$ is really a regular surface, we make the following straightforward computation.

$$
\begin{aligned}
\frac{\partial \mathbf{r}_{2}}{\partial s} & =\alpha(s)+\delta \frac{\partial}{\partial s}[\mathbf{n}(s)+\mathbf{n}(s) \times \boldsymbol{\alpha}(s) \cos t+\mathbf{n}(s) \sin t] \\
\frac{\partial \mathbf{r}_{2}}{\partial t} & =\delta[-\mathbf{n}(s) \times \boldsymbol{\alpha}(s) \sin t+\mathbf{n}(s) \cos t] \\
d \frac{\partial \mathbf{r}_{2}}{\partial s} \times \frac{\partial \mathbf{r}_{2}}{\partial t}= & \delta \boldsymbol{\alpha}(s) \times[-\mathbf{n}(s) \times \alpha(s) \sin t+\mathbf{n}(s) \cos t] \\
& +\delta^{2}\left(\frac{\partial}{\partial s}[\mathbf{n}(s)+\mathbf{n}(s) \times \boldsymbol{\alpha}(s) \cos t+\mathbf{n}(s) \sin t]\right) \\
& \times[-\mathbf{n}(s) \times \boldsymbol{\alpha}(s) \sin t+\mathbf{n}(s) \cos t] \\
= & \delta[-\mathbf{n}(s) \sin t+\boldsymbol{\alpha}(s) \times \mathbf{n}(s) \cos t]+\delta^{2} \mathbf{m}(s, t)
\end{aligned}
$$


where

$$
\begin{aligned}
\mathbf{m}(s, t)=\left(\frac{\partial}{\partial s}[\mathbf{n}(s)+\mathbf{n}(s) \times \boldsymbol{\alpha}(s) \cos t+\mathbf{n}(s) \sin t]\right) & \\
\times & {[-\mathbf{n}(s) \times \boldsymbol{\alpha}(s) \sin t+\mathbf{n}(s) \cos t] . }
\end{aligned}
$$

Then we have

$$
\begin{aligned}
\left|\frac{\partial \mathbf{r}_{2}}{\partial s} \times \frac{\partial \mathbf{r}_{2}}{\partial t}\right|^{2} & =\delta^{2}+2 \delta^{3}[-\mathbf{n}(s) \sin t+\boldsymbol{\alpha}(s) \times \mathbf{n}(s) \cos t] \cdot \mathbf{m}(s, t)+\delta^{4} \mathbf{m}^{2}(s, t) \\
& =\delta^{2}\left[1+\delta f(s, t)+\delta^{2} g(s, t)\right]
\end{aligned}
$$

where

$$
f(s, t)=2[-\mathbf{n}(s) \sin t+\alpha(s) \times \mathbf{n}(s) \cos t] \cdot \mathbf{m}(s, t), g(s, t)=\mathbf{m}^{2}(s, t) .
$$

Since $C$ is closed, the functions $f(s, t)$ and $g(s, t)$ are all bounded, thus there is a small positive number $\delta$ such that

$$
\left|\frac{\partial \mathbf{r}_{2}}{\partial s} \times \frac{\partial \mathbf{r}_{2}}{\partial t}\right|^{2}>\frac{1}{2} \delta^{2}>0
$$

Consequently, $S_{2}$ is a torus, that is, a closed, oriented surface of genus 1 .

Since $C$ is a curve on $S_{2}$ with $t=3 \pi / 2$, we have

$$
\left.\frac{1}{\delta}\left(\frac{\partial \mathbf{r}_{2}}{\partial s} \times \frac{\partial \mathbf{r}_{2}}{\partial t}\right)\right|_{t=(3 \pi / 2)}=\frac{1}{\delta} \boldsymbol{\alpha}(s) \times[\delta \mathbf{n}(s) \times \boldsymbol{\alpha}(s)]=\mathbf{n}(s),
$$

which implies that $\mathbf{n}(s)$ is the normal vector of $S_{2}$ along $C$. Since from above we know that $\mathbf{n}(s)$ is the normal vector of $S_{1}$ along $C$, too, the two sufaces $S_{1}$ and $S_{2}$ are tangent with each other along $C$. According to Joachimsthal's theorem, $C$ must be the line of curvature on $S_{2}$, for it is on $S_{1}$. The proof of Theorem 1 is completed.

Proof of Theorem 2: Let $M$ be an ovaloid, that is, a closed surface with positive Gauss curvature. If $C$ is a line of curvature on $M$, then as in the proof of Theorem 1, we have $\tau=-\dot{\theta}$ and

$$
T=-\int_{0}^{L} \dot{\theta}=\theta(0)-\theta(L) .
$$

Because the Gauss curvature of $M$ is positive, two principal curvatures $k_{1}$ and $k_{2}$ of $M$ are either all positive or all negative. Without loss of generality, we may choose the unit normal vector field $\mathbf{n}$ of $M$ in the outward direction of $M$ and so $k_{1}$ and $k_{2}$ are both negative, then by Euler's theorem, the normal curvature $k_{n}$ in the direction 
of $C$ is always negative, that is, $k_{n}<0$. Moreover, by Meusnier's theorem, we have $\kappa \cos \theta=k_{n}$, where $\kappa$ is the curvature of $C$ and always positive, thus we know that $\cos \theta=k_{n} / \kappa<0$, consequently, we obtain

$$
\theta(s) \in\left(\frac{\pi}{2}+2 m \pi, \frac{3 \pi}{2}+2 m \pi\right)
$$

where $m$ is an integer.

Since $M$ is a closed, oriented surface, we have, as in the proof of Theorem 1 , $\alpha(0)=\alpha(L)$ and $\beta(0)=\beta(L)$, and also $\mathbf{n}(0)=\mathbf{n}(L)$. Then we may obtain

$$
\theta(0)-\theta(L)=0 \bmod (2 \pi)
$$

Thus, by the continuity of $\theta(s)$, we may finally obtain, combining (24) and (25),

$$
\theta(0)-\theta(L)=0
$$

which implies that $T=0$ by (23). The proof of Theorem 2 is completed.

\section{REFERENCES}

[1] W. Blaschke, Kreis und Kugel (Wlater de Gruyter and Co., Berlin, 1956).

[2] Y.F. Chen, 'A new proof of a theorem of total torsion and its generalization', (in Chinese), Fujian Shifan Daxue Xuebao Ziran Kexue Ban 3 (1987), 11-14.

[3] H. Geppert, 'Sopra una caratterzione della spera', Ann. Mat. Pura. Appl. 20 (1941), 59-66.

[4] S. Millman and D. Parker, Elements of differential geometry (Prentice-Hall, Inc., New Jersey, 1977).

[5] M. Spivak, A comprehensive introduction to differential geometry, Vol. III (Publish or Perish Inc., Wilmington, Del., 1979).

Department of Mathematics South China University of Technology

Guangzhou 510641

China

e-mail: mayaqin@scut.edu.cn
Department of Mathematics South China Normal University Guangzhou 510631 China

e-mail: lisj@scnu.edu.cn 